\title{
A Survey on Optimum Neighbour Discovery Techniques in Mobile Ad-hoc Networks
}

\author{
Priyanka Agrawal \\ M.Tech. Student, \\ Computer Science \\ Engineering Department \\ Vikrant Institute of \\ Technology and Management, \\ Indore(M.P.)
}

\author{
Anwar Sarkeja \\ Assistant Professor, \\ Computer Science \\ Engineering Department \\ Vikrant Institute of \\ Technology and Management, \\ Indore(M.P.)
}

\begin{abstract}
There are several issues which are facing by Mobile ad hoc network and mainly it is related to routing and quality of service (QOS), It is happing because of variable nature and limited recourse constraints for real time application. In such type of network, for data transmission effective routing is necessary where each node behaves as a router. So many schemes have been directed in routing to discover route, one of them is flooding. In flooding scheme data packets are moving in whole network infinitely which is consuming resources redundantly such as battery power, bandwidth and cause throughput mortification. On the basis of earlier work the new approach is proposed for controlling flooding operation which removes flooding of routing packets. The work of new approach is to dealing with the discovery of convenient and optimum neighbour of source and all nodes in the network.
\end{abstract}

\section{Keywords}

MANET, Flooding, Issues, Routing Protocols

\section{INTRODUCTION}

Several mobile devices combine together and form mobile ad-hoc network which is wireless. And there is no need of fixed infrastructure or centralized control. In network all devices are serving as router, which deliver data to other nodes. Applications of mobile ad hoc networks are battlefield area, meetings or conventions, emergency disaster relief, personnel coordinating efforts after an earthquake [1]. There are several issues which are facing by Mobile ad hoc network and mainly it is related to routing and quality of service (QOS), it is happing because of variable nature and limited recourse constraints for real time application. In such type of network, for data transmission effective routing is necessary where each node behaves as a router. So many schemes have been directed in routing to discover route, one of them is flooding. In flooding scheme data packets are moving in whole network infinitely which is consuming resources redundantly such as battery power, bandwidth and cause throughput mortification.

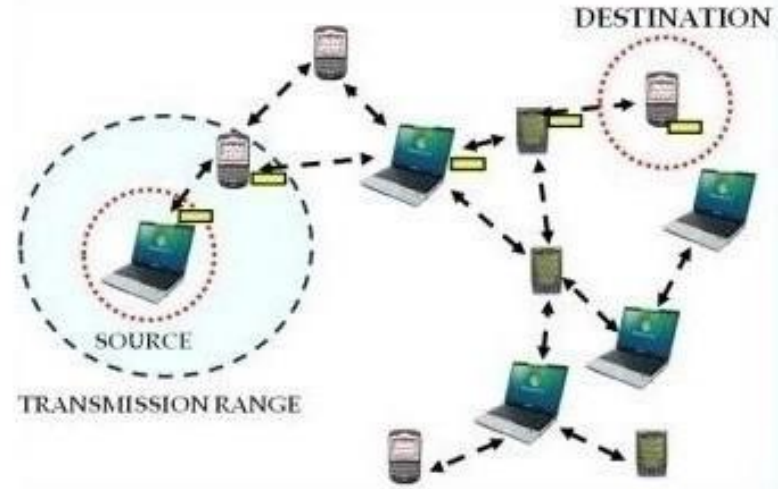

Figure 1 Mobile Ad-hoc Network

According to the mobile nature and lack of fixed infrastructure, MANET has included too many characteristics which are mention below:

Infrastructure less: MANET if self-coordinated and self ruling collection of mobile nodes. In which wireless links are the medium for the mobile devices for communication which does not have any connection and dependency on any preestablished infrastructure or central access point.

Autonomous Terminal: In MANET each and every mobile devices are self ruling devices and they can perform function of router as well as host communication freely [3].

Multi-hop Routing: In MANET there are two types of adhoc routing algorithms single hop and multi hop which is based on different routing protocols and link layer attributes.

Dynamic Network Topologies: Each mobile node is free to move promptly in MANET in any direction and quickly change its topology to other nodes in the network.

Energy Constrained Operation: Mobile devices are carrying battery power therefore they have limited power supply [3]. 
MANET Issues

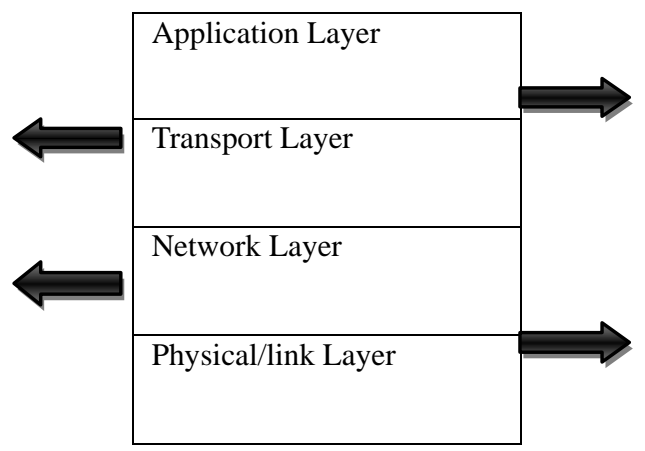

Figure 2 Issues of MANET

The main issues faced at different layers of MANET [5] are shown in figure 2. Layered architecture of ad-hoc network is given below:

Routing: Topology of network is frequently and constantly changing as well as communication is very challenging task between the nodes because mobile nodes of MANET are free to move randomly in any direction. To recover the path several routing protocols have been developed.

Limited wireless transmission range: Radio Bandwidth limitation is fixed for wireless networks and while comparing with wireless networks it can offer data rates much less. In maintaining topological information limited transmission rang affects routing protocols.

Packet losses due to transmission errors: Ad-hoc wireless network is suffering a packet loss problem because of high bit error rate in wireless channel, hidden terminal problem, location dependency, unidirectional link, path break etc.

Security and Reliability: In the network between mobile nodes security of connection needed by ad hoc network because of the feature of distributed operation. Malicious host finding it easy to bug during communication session and this could lead through illegal access, information break-in, conflict, jamming and service degradation. Wireless transmission range is limited so wireless links have reliability problem, the broadcast nature of wireless medium e.g. hidden terminal problem; mobility causes packet loss and data transmission errors.

Quality of Service: Due to invariably changing environment, providing quality of service will be difficult task. MANET has changing environment and during transmission it does not provide the guarantee of services.

Energy Constrained: Important criteria of system design are energy conversation because every node in the MANET may depend on batteries.

Limited Bandwidth: In the comparison of wired network wireless network have much lower capacity. Also because of the multiple access, fading noise and interference condition it's the wireless links which have lower throughout [6].

\section{BACKGROUND}

In MANETs flooding is a major operation. Most of the major routing protocols, like DSR [13], AODV [27], LAR [17], ZRP [10], etc., rely on flooding for propagating route discovery, route maintenance, or topology update packets. Flooding is usually start function in MANETs. So, that an efficient implementation of the flooding scheme is difficult for reducing the overhead of routing protocols and improving the throughput of networks. Efficient flooding schemes are different from the broadcast mechanisms discussed in [19][44]. Broadcast mechanism is being used in transmission of large amount of data or stream media data. These applications require an efficient broadcast route before the actual transmission of data, so that data can be transmitted efficiently along the pre-found route. In contrast, flooding is usually used in dissemination of control packets, which is a one-off operation and it does not need routing beforehand.

- $\quad$ Pure flooding

Pure flooding also called blind flooding, is the simplest flooding technique. The basic idea of this approach is every node in the network retransmits the flooding message when it is the first time to receive it [9] [12]. A node, on receiving a broadcast message for the first time, has the responsibility to rebroadcast the message. It costs $\mathrm{n}$ transmissions in a network with $\mathrm{n}$ nodes. This simple scheme guarantees that a flooding message can reach all nodes if the network is connected and there is no collision.

\section{- Probabilistic flooding scheme}

One approach to alleviate the broadcast storm problem is to inhibit some nodes from rebroadcast to reduce the redundancy, and thus contention and collision. Sze-Yao Ni et al. [26] presented a probabilistic scheme that use a probabilistic rebroadcasting and differentiate timing of rebroadcasts to avoid redundancy and collisions. The basic idea of probabilistic flooding schemes is that each node forwards a flooding message with probability $\mathrm{P}$ upon receiving it for the first time. Clearly, when $\mathrm{P}=1$, this scheme is equivalent to pure flooding. The probabilistic schemes can be classified into four types: counter-based, distance-based, location-based and cluster-based. These schemes differ in how a node estimates redundancy and how it accumulates knowledge to assist its decision. Except the last scheme, which relies on some local connectivity information, all schemes operate in a fully distributed manner.

- $\quad$ Flooding with Self Pruning (FSP)

The simplest flooding scheme based on 1-hop neighbour knowledge is flooding with self pruning (FSP) proposed by Lim and Kim [22]. FSP is a receiver-based scheme which uses 1-hop information. A sender forwards a flooding message by attaching all of its 1-hop neighbours to the message. A receiver compares its own 1-hop neighbours with the node list in the message; it will not

forwards the message if all its 1-hop neighbours are already included in the list, otherwise it forwards the message as a sender

\section{RELATED STUDY}

To reduce the flooding traffic, many approaches have been proposed [7].

0-hop schemes: Many flooding techniques developed in early time are in this category. These techniques try to reduce flooding cost without any assumption on neighbourhood knowledge. For example, one can simply make each host rebroadcast flooding packets with some predetermined probability. This probabilistic-based scheme was first proposed in [8] [9] and further investigated in [10].

1-hop schemes: The technique called Flooding with Self Pruning (FSP) in [11] is a 1-hop flood- ing scheme since it requires each host to track its neighbours within 1-hop distance. In this scheme, when a host broadcasts a packet, it includes all of its 1-hop neighbours in the packet header. 
Upon receiving a broadcast, a host checks its own 1-hop neighbours and if all of them have already been listed in the broadcast packet header, it does not forward the broadcast.

2+ -hop schemes: Most existing flooding approaches are in this category and they can be further divided into reactive schemes and proactive schemes. In proactive schemes [11] [12] [13] [14], abroad casting host selects some of its 1-hop neighbours as rebroadcasting hosts. When a host receives a broadcast, it drops off the packet if it is not designated as a rebroadcasting host; otherwise, it recursively chooses some of its 1-hop neighbours as rebroadcasting hosts and then forwards the broadcast.

\section{PROPOSED METHDOLOGY}

Routing is essential operation in the mobile ad-hoc network for data transmissions because every node serves as router in absence of central router. Several routing schemes have been advised to discover route, one of them is flooding. Flooding scheme offers to discover route through flooding of routing packets such as route request (RREQ) in the network. In this scheme routing packets are traversing throughout the network infinitely that consumes resources unnecessarily such battery power, bandwidth and cause throughput degradation.

To remove flooding of routing packets in mobile ad-hoc network, an approach is proposed on the basis of earlier approach which control flooding operation. Proposed approach deals with the discovery of suitable or optimum neighbour of source and further downstream nodes in the network. For discovery of suitable nodes, an approach determines mobility factor, energy and receiver gain of nodes.

Network simulator tool such as NS-2 is required which helps to simulate the mentioned objectives and analysis of network performance considering different parameters.

\section{Network Simulator (NS)}

Network Simulator is a discrete event simulator for networking research. NS supply widespread support for simulation of TCP, routing protocols over wired and wireless (local and satellite) networks. A simulator model a real-world system is essentially a simplification of the real-world system itself. This explanation describes some of the boundaries of the simulation model personified in the current release of NS-2.

The general process of a simulation can be divided into several steps:

- Topology definition: To ease the formation of basic conveniences and define their interrelationships, NS2 has a system of helpers that facilitates this process.

- Model usage: Models are additional facility to simulation like UDP, IPv4, point-to-point devices, links and applications. Mostly this is done with helpers.

- Node and link configuration: Models set their default values for example, the size of packets sent or MTU of a point-to-point link; most of the time this is done using the attribute system.

- Execution: Simulation facilities make events; data demand by the user is logged.
- Performance analysis: When the simulation is ended and data are available as a time-stamped event traces. Then this data can then be analyzed with tools like $\mathrm{R}$ to draw conclusions.

- Graphical Visualization: Processed data gather in a simulation can be visualized using graph with tools like Gnuplot, matplotlib or Xgraph. Xgraph.These is the plotting tool bundled with installation packages.

\section{REFERENCES}

[1] Talha, "Improving Performance of Mobile Ad-hoc Networks using Efficient Tactical On - Demand Distance Vector (TAODV) Routing Algorithm," proceeding of International Journal of Innovative Computing, Information and Control, Volume 8, November 6, June 2012 ISSN: 1349- 4198, pp 43754389 .

[2] Dr. S. S. Dhenakaran and A. Parvathavarthini, "An Overview of Routing Protocols in Mobile Ad-hoc Network," proceeding of International Journal of Advanced Research in Computer Science and Software Engineering, Volume 3, Issue 2, February 2013, ISSN: 2277-128X, pp. 251-259.

[3] Imrich Chlamtac, Marco Conti and Jennifer J. N. Liu, "Mobile Ad-hoc Networking: Imperatives and Challenges," proceeding of Science Direct Ad-hoc Networks 1, (2003) pp. 13-64.

[4] Mohit Kumar and Rashmi Mishra, "An overview of MANET: History, Challenges and Applications," proceeding of Indian Journal of Computer Science and Engineering, Volumes 3, No. 1, Feb-Mar 2012, ISSN: 0976-5166, pp. 121-125.

[5] Sunil Taneja and Ashwani Kush, "A Survey of Routing Protocols in Mobile Ad-hoc Networks," proceeding of International Journal of Innovation, Management and Technology, Volume 1, No. 3, August 2010, ISSN: 2010-0248, pp. 279-285.

[6] G. S. Mamatha and Dr. S C Sharma, "Analyzing the MANET Variations, Challenges Capacity and Protocol Issues," proceeding of the International Journal of, Computer Science and Engineering Survey (IJCSES) Volume 1, No. 1, August 2010, pp. 14-21.

[7] Ying Cai, Kien A. Hua, Aaron Phillips ,'Leveraging 1hop Neighbourhood Knowledge for Efficient Flooding in Wireless Ad Hoc Networks"

[8] S. Ni, Y. Tseng, Y. Chen, and J. Sheu. The Broadcast Storm Problem in a Mobile Ad Hoc Network. In Proc. Of MOBICOM'99, pages 151-162, 1999.

[9] Y. Tseng, S. Ni, and E. Y. Shih. Adaptive Approaches to Relieving Broadcast Storms in a Wireless Multihop Mobile Ad Hoc Networks. In Proc. of ICDCS'01, pages 481-488, 2001.

[10] Y. Sasson, D. Cavin, and A. Schiper. Probabilistic broad-cast for flooding in wireless mobile ad hoc networks. In Swiss Federal Institute of Technology, Technical Report IC/2002/54, 2002. 
[11] H. Lim and C. Kim. Multicast Tree Construction and Flooding in Wireless Ad Hoc Networks. In Proc. of the ACM Int'l Workshop on Modeling, Analysis and Simulation of Wireless and Mobile Systems (MSWIM), pages 61-68, August 2000.

[12] W. Lou and J. Wu. On Reducing Broadcast Redundancy in Ad Hoc Wireless Networks. IEEE Transactions on Mobile Computing, 1(2):111-123, 2002.
[13] W. Peng and X. Lu. AHBP: An Efficient Broadcast Protocol for Mobile Ad Hoc Networks. Journal of Science and Technology, Beijing, China, 2002.

[14] A. Laouiti, A. Qayyum, and L. Viennot. Multipoint relaying: An efficient technique for flooding in mobile wireless networks. In 35th Annual Hawaii International Confer- ence on System Sciences (HICSS'2001). IEEE Computer Society, 2001. 